\title{
Supercurrent flow through the network of spin-vortices in cuprates
}

\author{
Hiroyasu Koizumi, Akira Okazaki \\ Division of Materials Science, University of Tsukuba,Tsukuba, Ibaraki 305-8573, Japan* \\ Michel Abou Ghantous \\ Department of Physics, Texas A \& $M$ University at Qatar, \\ Education City, PO Box 23874, Doha, Qatar \\ Masashi Tachiki \\ Department of Physics, Tohoku University, Sendai, Miyagi 980-8578, Japan
}

(Dated: November 13, 2014)

\begin{abstract}
We propose a novel supercurrent generation mechanism in the cuprate. The supercurrent is generated as a collection of the spin-vortex-induced loop currents created with the doped holes at their centers. A quartet of the spin-vortices with width $4 a$ ( $a$ is the lattice constant of the $\mathrm{CuO}_{2}$ plane) is the stable unit of the spin-vortices, and an assembly of them create a network channel for the supercurrent flow. A macroscopic supercurrent flows when they cover the whole $\mathrm{CuO}_{2}$ plane. The Ginzburg-Landau macroscopic wave function formalism is also derived from the present supercurrent generation mechanism.
\end{abstract}

PACS numbers:

*koizumi@ims.tsukuba.ac.jp 


\section{INTRODUCTION}

More than a quarter of a century has passed since the high temperature superconductivity was found in the hole doped cuprate [1]. As the origin of the high temperature superconductivity, the electron spin degree of freedom is expected to play an important role as is manifested by the fact that the superconductivity occurs in the vicinity of the antiferromagnetic Mott insulating phase. In this respect, it is notable that the 'hourglass-shaped magnetic excitation spectrum' indicates the presence of a peculiar spin texture in the cuprate [2]. As the origin for it, the stripe model is a popular explanation [3]. However, another possibility is the spin-vortex model [4-6]. In this model, the spin-vortices are created by itinerant electrons in the $\mathrm{CuO}_{2}$ plane; the doped holes form small polarons by the strong hole-lattice interaction at low temperatures and become the cores of the spin-vortices; the holes are expected to provide a superexchange interaction between spins across them, and stabilize the spin-vortices [7].

It has been shown that the spin-vortex model also explains other phenomena observed in the cuprate such as 1) nonzero Kerr rotation in zero-magnetic field after exposed in a strong magnetic field [8]; 2) the change of the sign of the Hall coefficient with temperature change [9]; 3) the suppression of superconductivity in the $\mathrm{x}=1 / 8$ static-stripe ordered sample [10]; 4) a large anomalous Nernst signal, including its sign-change with temperature change $[11,12] ; 5)$ the 'Fermi-arc' in the ARPES [13]; 6) the change of the $\mathrm{Cu}-\mathrm{O}$ bond length by doping $[14,15]$.

In the spin-vortex model, the appearance of a loop current called, the 'spin-vortex-induced loop current (SVILC)' is predicted [4]. The size of the SVILC is in the order of the lattice constant of the $\mathrm{CuO}_{2}$ plane, similar to the coherence length of the superconductivity. The SVILC persists as long as the spin-vortex induces it persists; due to this stability, a single SVILC is considered as a localized supercurrent of the coherence length size. A macroscopic supercurrent is generated as a collection of them, and it explains the flux quantum $c h / 2 e$ $[4-7]$.

In the cuprate, the relation between the appearance of the superconductivity and the Cooper pair formation does not follow the BCS-Eliashberg theory; the superconducting transition temperature $T_{\mathrm{c}}$ is determined by the fluctuation of the supercurrent vortex of the coherence length size [16]. Although the Cooper pair formation is indicated by the ARPES 
spectrum in the nodal region [17], the 'protagonist' of the supercurrent generation may be the supercurrent vortex of the coherence length size appeared in the estimate of $T_{\mathrm{c}}$. The recent atomic-scale STM study [18] indicates that the macroscopic superconductivity is realized through the interconnection of unidirectional clusters of doped holes with width $4 a$, where $a$ is the lattice constant of the $\mathrm{CuO}_{2}$ plane. Then, the observed enhanced Nernst effect in the pseudogap phase [11] may be attributed to the localized supercurrent in these clusters, and the global superconductivity is realized by the interconnection of them. Actually, this behavior is naturally explained by the supercurrent generation mechanism of the spin-vortex model $[4,7]$. In the present work, we propose a novel mechanism of the supercurrent generation in the cuprate based on the spin-vortex model: the localized superconductivity occurs due to the formation of a quartet of spin-vortices (we call it the 'spin-vortex quartet' (SVQ)), and the macroscopic supercurrent flow occurs when an assembly of the SVQs creates a network that covers the whole $\mathrm{CuO}_{2}$ plane.

The organization of the present work is following: in Section II, the Hamiltonian we use for the cuprate superconductivity is given. In Section III, a way to solve the Schrödinger equation for the Hamiltonian given in Section II is presented by imposing the single-valued requirement of wave functions in the presence of spin-vortices. In Section IV, calculations for the supercurrent generated as a collection of SVILCs are performed, where a network of the SVQs provide a channel for the supercurrent. In Section V, we derive the Ginzburg-Landau type equation from the spin vortex theory. Lastly, we conclude the present work in Section VI.

\section{THE MODEL HAMILTONIAN FOR THE CUPRATE SUPERCONDUCTOR AND THE APPEARANCE OF THE SPIN-TWISTING MOTION OF ITINERANT ELECTRONS}

Our model Hamiltonian for electrons in the $\mathrm{CuO}_{2}$ plane of the cuprate is given by

$$
H_{C u O_{2}}=-\sum_{\langle i, j\rangle_{1}, \sigma} t\left(c_{i \sigma}^{\dagger} c_{j \sigma}+c_{j \sigma}^{\dagger} c_{i \sigma}\right)+U \sum_{j} c_{j \uparrow}^{\dagger} c_{j \uparrow} c_{j \downarrow}^{\dagger} c_{j \downarrow}+H_{\mathrm{h}-1}
$$

where $i$ and $j$ are sites in the two-dimensional square lattice in the $\mathrm{CuO}_{2}$ plane; the coppers reside at the lattice points and the oxygens that exist between nearest neighbor coppers are not explicitly taken into account; $\langle i, j\rangle_{1}$ indicates the nearest neighbor pairs; $c_{j \sigma}^{\dagger}$ and $c_{j \sigma}$ are 
the creation and annihilation operators of electrons at the $j$ th site with the $z$-axis projection of electron spin $\sigma$, respectively. The term $H_{\mathrm{h}-1}$ is the hole lattice interaction.

We further simplify the problem by employing the extreme limit where the doped holes are immobile at temperatures below $T_{c}$. This assumption will be justified since the experiment [14] and theory [15] indicate that the holes form small polarons at low temperatures. Then, the system is in the effectively half-filled situation (EHFS) where the number of electrons and that of the accessible sites are equal. One might think that the current flow is impossible in this situation since the value of $U / t$ is large for the cuprate, thus, the half-filled insulating state will be realized. However, the forced whole system motion generated by the singlevalued requirement of wave functions gives rise to the spin-vortex-induced loop currents (SVILCs)[7]. We consider the supercurrent generation by these SVILCs in this work.

In the EHFS, Eq. (1) is rewritten as,

$$
H_{\mathrm{EHFS}}=-\sum_{\langle i, j\rangle_{1}, \sigma} t\left(c_{i \sigma}^{\dagger} c_{j \sigma}+c_{j \sigma}^{\dagger} c_{i \sigma}\right)+U \sum_{j} c_{j \uparrow}^{\dagger} c_{j \uparrow} c_{j \downarrow}^{\dagger} c_{j \downarrow}
$$

where the sum $i$ and $j$ are taken over only the accessible sites of electrons, i.e., the sites occupied by the holes are omitted. We adopt this convention for the site indices $i, j, k$, and $\ell$, below.

In addition to $H_{\mathrm{EHFS}}$ we add the following spin exchange interaction between spins across the hole occupied sites,

$$
H_{s v}=J^{\prime} \sum_{\langle i, j\rangle_{h}} \hat{\mathbf{S}}_{i} \cdot \hat{\mathbf{S}}_{j}
$$

where $\hat{\mathbf{S}}_{j}$ is the spin moment operator at the $j$ th site given by

$$
\hat{\mathbf{S}}_{j}=\frac{1}{2} \sum_{\sigma, \sigma^{\prime}} c_{j \sigma}^{\dagger} \boldsymbol{\sigma}_{\sigma \sigma^{\prime}} c_{j \sigma^{\prime}}
$$

$\boldsymbol{\sigma}$ is the vector of Pauli matrices, $\langle i, j\rangle_{h}$ indicates the pairs across the hole occupied sites; it includes also pairs of sites for which the sites $i$ and $j$ are in the right angle positions with respect to the hole occupied site. The above interaction stabilizes the spin-vortices formed around the doped holes, thus, this term is crucial for the spin-vortex model.

The value of $J^{\prime}$ is expected to be large in the cuprate due to the small polaron formation around the doped hole: the molecular orbital cluster calculation result indicates that when the small polaron is formed, the copper $d_{x^{2}-y^{2}}$ and the surrounding four oxygen $p_{\sigma}$ orbitals 
form a molecular (or hybridized) orbital [15]. Then, the exchange parameter $J^{\prime}$ across the hole occupied sites is calculated by treating the hole molecular orbital as the intermediate level for the perturbation calculation [7, 19];

$$
J^{\prime} \approx \frac{4 t_{d h}^{4}}{\left(\varepsilon_{h}-\varepsilon_{d}\right)^{3}}
$$

where the parameter $t_{d h}$ is the transfer integral between the spin-reside copper $d_{x^{2}-y^{2}}$ orbital and the hole orbital $h$ at the hole-occupied site; $\varepsilon_{d}$ and $\varepsilon_{h}$ are the orbital energies of $d_{x^{2}-y^{2}}$ and $h$, respectively. Since the hopping integral $t_{d h}$ is not so much different from that between the copper $d_{x^{2}-y^{2}}$ orbital and the nearby oxygen $p_{\sigma}$ orbital, and the energy difference $\varepsilon_{h}-\varepsilon_{d}$ is sufficiently small, $J^{\prime}$ is expected to be in the order of $J$, where $J=4 t^{2} / U$. We use the value of $J^{\prime}=0.5 J$ in the later calculation.

The spin-vortex is characterized by the topological winding number. Although the spin configuration fluctuates temporarily and also quantum mechanically, the winding number is expected to be robust since it is topological integer. The winding number of the spin-vortex is calculated using the spin moment $\mathbf{S}_{j}=\left(S_{j}^{x}, S_{j}^{y}, S_{j}^{z}\right)$; its components are expressed by the azimuth angle $\xi$ and polar angle $\zeta$

$$
\begin{aligned}
& S_{j}^{x}=\frac{1}{2}\left\langle c_{j \uparrow}^{\dagger} c_{j \downarrow}+c_{j \downarrow}^{\dagger} c_{j \uparrow}\right\rangle=S_{j} \cos \xi_{j} \sin \zeta_{j} \\
& S_{j}^{y}=-\frac{i}{2}\left\langle c_{j \uparrow}^{\dagger} c_{j \downarrow}-c_{j \downarrow}^{\dagger} c_{j \uparrow}\right\rangle=S_{j} \sin \xi_{j} \sin \zeta_{j} \\
& S_{j}^{z}=\frac{1}{2}\left\langle c_{j \uparrow}^{\dagger} c_{j \uparrow}-c_{j \downarrow}^{\dagger} c_{j \downarrow}\right\rangle=S_{j} \cos \zeta_{j}
\end{aligned}
$$

where the subscript $j$ indicates the $j$ th site in the lattice whose coordinate vector is $\mathbf{r}_{j} ;\langle\hat{O}\rangle$ denotes the expectation value of the operator $\hat{O}$. We may take $\zeta=\pi / 2$ for all the sites (actually, the numerical calculation in Section IV yields this value); then, the spins are lying in the $\mathrm{CuO}_{2}$ plane, and the background antiferromagnetic spin is given by $\xi_{j}=\pi\left(j_{x}+j_{y}\right)$, where $\left(j_{x}, j_{y}\right)$ is the $x-y$ coordinates of the $j$ th site taking the lattice constant $a=1$. We define $\eta_{j}$ as

$$
\eta_{j}=\xi_{j}-\pi\left(j_{x}+j_{y}\right)
$$

and separate the background antiferromagnetic contribution. The winding number of $\eta$ for loop $C_{\ell}$ is defined as

$$
w_{\ell}[\eta]=\frac{1}{2 \pi} \sum_{i=1}^{N_{\ell}}\left(\eta_{C_{\ell}(i+1)}-\eta_{C_{\ell}(i)}\right)
$$


where $C_{\ell}$ is a loop in the $x-y$ plane. $N_{\ell}$ is the total number of sites on the loop $C_{\ell}$, and $C_{\ell}(i)$ is the $i$ th site on it with the periodic condition $C_{\ell}\left(N_{\ell}+1\right)=C_{\ell}(1)$. In Fig. 1b, spin-vortices embedded in the antiferromagnic background given in Fig. 1a are depicted, where a center of the spin-vortex with winding number +1 is denoted as ' $\mathrm{M}$ ', and that of -1 as ' $\mathrm{A}$ '. In this figure, the $\mathrm{CuO}_{2}$ plane is approximated as a two-dimensional square lattice by only retaining copper sites without explicitly taking into account the oxygens that exist between the nearest coppers.

In the spin-vortex model, the spin-vortices are generated by itinerant electrons. In this situation, the electrons move in the Hartree-Fock field that contains an effective magnetic field of the exchange type created by the spin-vortices. Due to the effective magnetic field, the electrons move with twisting their spin directions [7].

The effective magnetic field arises from the on-site Coulomb repulsion term in Eq. (1) given by

$$
\sum_{j} U c_{j \uparrow}^{\dagger} c_{j \uparrow} c_{j \downarrow}^{\dagger} c_{j \downarrow}=\sum_{j} U\left(\frac{\hat{n}_{j}}{2}-\frac{2}{3} \hat{\mathbf{S}}_{j}^{2}\right) \approx \frac{U N}{2}-\frac{2}{3} \sum_{j} U\left(2 \mathbf{S}_{j} \cdot \hat{\mathbf{S}}_{j}-\mathbf{S}_{j}^{2}\right)
$$

where $\hat{n}_{j}$ is the number operator at the $j$ th site given by

$$
\hat{n}_{j}=c_{j \uparrow}^{\dagger} c_{j \uparrow}+c_{j \downarrow}^{\dagger} c_{j \downarrow}
$$

and $N$ is the number of electrons. The effective magnetic field that acts on the spin of electron appears if $\mathbf{S}_{j} \neq 0$. If the spin-vortices exist, circular change of the effective field along loops in the coordinate space occurs. The electron motion then becomes the one with twisting its spin direction.

The electronic basis functions that diagonalize Eq. (9) are given by

$$
\begin{aligned}
& \left|-\left[\mathbf{r}_{j}\right]\right\rangle=e^{-i \frac{\chi_{j}}{2}}\left(\cos \frac{\zeta_{j}}{2} e^{-i \frac{\xi_{j}}{2}} c_{j \downarrow}^{\dagger}+\sin \frac{\zeta_{j}}{2} e^{i \frac{\xi_{j}}{2}} c_{j \uparrow}^{\dagger}\right)|\mathrm{vac}\rangle \\
& \left|+\left[\mathbf{r}_{j}\right]\right\rangle=e^{-i \frac{\chi_{j}}{2}}\left(-\sin \frac{\zeta_{j}}{2} e^{-i \frac{\xi_{j}}{2}} c_{j \downarrow}^{\dagger}+\cos \frac{\zeta_{j}}{2} e^{i \frac{\xi_{j}}{2}} c_{j \uparrow}^{\dagger}\right)|\mathrm{vac}\rangle
\end{aligned}
$$

where $|v a c\rangle$ denotes the vacuum state. The salient feature of the above basis functions is that in contrast to the basis $\left\{c_{j \uparrow}^{\dagger}|\mathrm{vac}\rangle, c_{j \downarrow}^{\dagger}|\mathrm{vac}\rangle\right\}$, they are zero at the centers of the spin-vortices to be consistent with the fact that the value of $\xi$ is not defined there. Another notable point is that angular variable $\chi_{j}$ of period $2 \pi$ is added to make the basis functions single-valued with respect to $\mathbf{r}_{j}$ [7]. In other words, the above basis takes into account the singularities 
a

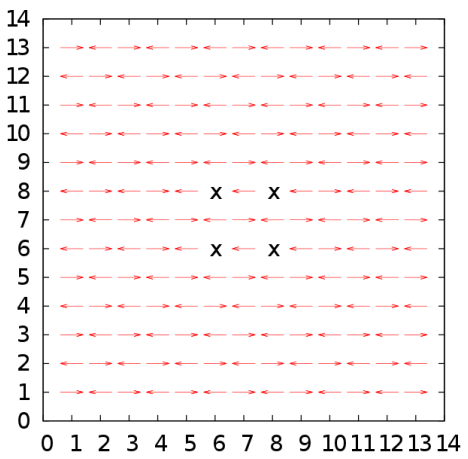

d

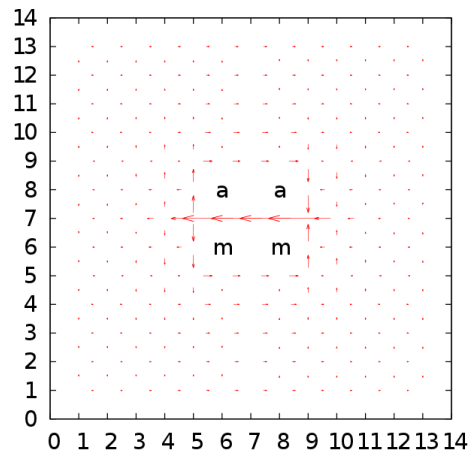

b

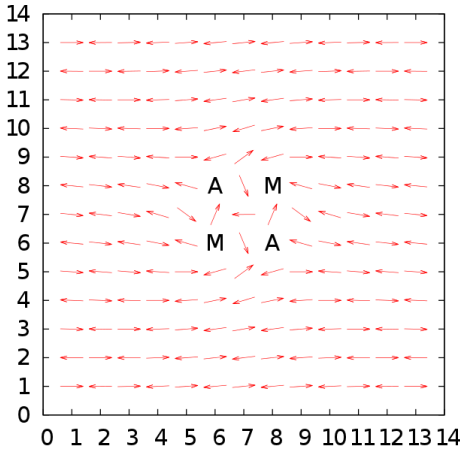

e

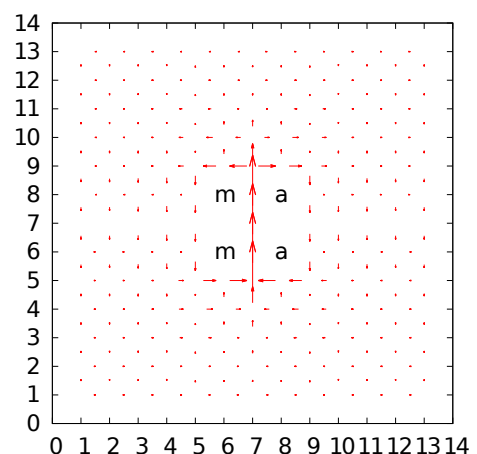

C
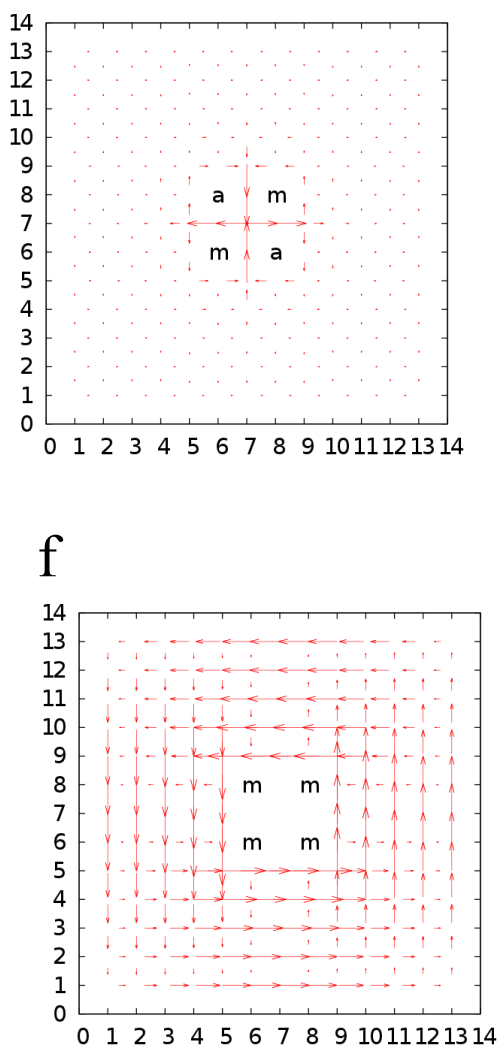

FIG. 1: Plots of the spin configuration and current pattern for the system with four holes in the $13 \times 13$ two-dimensional square lattice. a, Spin configuration for the antiferromagnetic $(\mathrm{AF})$ case. ' $\mathrm{X}$ ' denote the position of a hole. $\mathbf{b}$, Spin configuration with one spin-vortex quartet (SVQ). ' $M$ ' and ' $A$ ' indicate the centers of the winding number +1 and -1 spin-vortices, respectively. $\mathbf{c}$, The minimal energy current distribution. ' $m$ ' and ' $a$ ' indicate the centers of the winding number +1 and -1 SVILCs, respectively. This state is higher in energy than the AF case by $1.39 t$ if the term in Eq. (3) is absent; however, lower by $0.21 t$ if $J^{\prime}=0.5 J$ where $J=4 t^{2} / U, U=8 t$ is employed in Eq. (3). d, The second minimal energy current distribution. This state is higher in energy than c by $0.44 t$. e, The second minimal energy current distribution. This state is degenerate with the state in $\mathbf{d}$. f, The maximum energy current distribution. This state is higher in energy than $\mathbf{c}$ by $6.52 t$.

of the wave function arising from the spin-vortices. It is known that such singularities give rise to a Berry phase [20] or an Aharonov-Bohm type effect by the gauge potential for the 
fictitious magnetic field,

$$
\mathbf{A}^{\text {fic }}=\frac{\hbar c}{2 q} \nabla \chi
$$

where $q=-e[7]$. The use of the basis $\left\{\left|-\left[\mathbf{r}_{j}\right]\right\rangle,\left|+\left[\mathbf{r}_{j}\right]\right\rangle\right\}$, instead of $\left\{c_{j \uparrow}^{\dagger}|\operatorname{vac}\rangle, c_{j \downarrow}^{\dagger}|\operatorname{vac}\rangle\right\}$, includes the Berry phase effect due to the twisting spin motion of the electron in the presence of the spin-vortices.

Let us explain the necessity of the phase factor $e^{-i \frac{\chi_{j}}{2}}$. If a spin-vortex exist, the phase shift of $\xi$ after a circular transportation along the loop $C_{\ell}$ in the coordinate space is $2 \pi w_{\ell}[\xi] \neq 0$, where $w_{\ell}[\xi]$ is the winding number of $\xi$ calculated by replacing $\eta$ in Eq. (8) with $\xi$. Then, $\xi_{j}$ exhibits the jump of value $\xi_{j} \rightarrow \xi_{j}+2 \pi w_{\ell}[\xi]$ after the excursion along $C_{\ell}$, where the site $j$ is on $C_{\ell}$. If $w_{\ell}[\xi]$ is odd, the sign-change occurs for the phase factors $e^{ \pm i \frac{\xi_{j}}{2}}$ as

$$
e^{ \pm i \frac{\xi_{j}}{2}} \rightarrow e^{ \pm i \frac{\xi_{j}+2 \pi w_{\ell}[\xi]}{2}}=-e^{ \pm i \frac{\xi_{j}}{2}}
$$

The angular variable $\chi$ is so chosen that the phase factor $e^{-i \frac{\chi_{j}}{2}}$ compensates this sign-change.

The condition for $\chi$ that makes $\left|-\left[\mathbf{r}_{j}\right]\right\rangle$ and $\left|+\left[\mathbf{r}_{j}\right]\right\rangle$ single-valued is

$$
w_{\ell}[\xi]+w_{\ell}[\chi]=\text { even number for any loop } C_{\ell}
$$

where $w_{\ell}[\chi]$ is the winding number of $\chi$ calculated by replacing $\eta$ in Eq. (8) by $\chi$. The winding number of $\xi, w_{\ell}[\xi]$, in the above condition can be replaced by that of $\eta, w_{\ell}[\eta]$. If the above condition is satisfied, the phase change of $\pm \xi_{j}-\chi_{j}$ is a multiple of $4 \pi$ after the circular transportation along $C_{\ell}$. Consequently, the eigenfunctions $\left|+\left[\mathbf{r}_{j}\right]\right\rangle$ and $\left|-\left[\mathbf{r}_{j}\right]\right\rangle$ become single-valued.

The condition in Eq. (14) is not sufficient to specify $\chi$; for example we may adopt $\chi=\xi$ or $\chi=-\xi$, or something else. The way to determine the angular variable $\chi$ will be explained in the next section.

The crucial point to obtain states with the spin-vortex-induced loop current is to express the single-particle wave function as

$$
|\gamma\rangle=\sum_{j}\left(D_{j-}^{\gamma}\left|-\left[\mathbf{r}_{j}\right]\right\rangle+D_{j+}^{\gamma}\left|+\left[\mathbf{r}_{j}\right]\right\rangle\right)
$$

where $D_{j-}^{\gamma}$ and $D_{j+}^{\gamma}$ are parameters obtained by the numerical evaluation of the wave function. This is a single-valued wave function for itinerant electrons with spin-twisting motion. 


\section{SELF-CONSISTENT FIELD CALCULATION WITH INCLUDING THE SINGLE-VALUEDNESS CONSTRAINT OF WAVE FUNCTIONS}

We will describe the way we solved the Schrödinger equation given in Section II with including the single-valuedness constraint of wave functions. The method described below is actually an improved version presented in Ref. [7].

Let us consider the Hamiltonian $H_{\mathrm{EHFS}}$ in Eq. (2). We use the following Hartree-Fock Hamiltonian for it [21],

$$
\begin{aligned}
H_{\mathrm{EHFS}}^{H F} & =-t \sum_{\langle i, j\rangle_{1}, \sigma}\left(c_{i \sigma}^{\dagger} c_{j \sigma}+c_{j \sigma}^{\dagger} c_{i \sigma}\right) \\
+ & U \sum_{j}\left[\left(\frac{n_{j}}{2}-S_{j}^{z}\right) c_{j \uparrow}^{\dagger} c_{j \uparrow}+\left(\frac{n_{j}}{2}+S_{j}^{z}\right) c_{j \downarrow}^{\dagger} c_{j \downarrow}-\left(S_{j}^{x}-i S_{j}^{y}\right) c_{j \uparrow}^{\dagger} c_{j \downarrow}-\left(S_{j}^{x}+i S_{j}^{y}\right) c_{j \downarrow}^{\dagger} c_{j \uparrow}\right]
\end{aligned}
$$

where $n_{j}=\left\langle\hat{n}_{j}\right\rangle$ is the number density of electrons at the $j$ th site.

By self-consistently diagonalizing $H_{\mathrm{EHFS}}^{H F}$ in Eq. (??), we obtain the following Hartree-Fock orbitals;

$$
|\tilde{\gamma}\rangle=\sum_{j}\left[\tilde{D}_{j \uparrow}^{\gamma} c_{j \uparrow}^{\dagger}+\tilde{D}_{j \downarrow}^{\gamma} c_{j \downarrow}^{\dagger}\right]|\operatorname{vac}\rangle
$$

where $\tilde{D}_{j \uparrow}^{\gamma}$ and $\tilde{D}_{j \downarrow}^{\gamma}$ are numerical parameters obtained from the diagonalization. By constructing the Slater determinant with the occupied orbitals, the Hartree-Fock wave function is obtained, and the self-consistent spin moment $\mathbf{S}_{j}=\left(S_{j}^{x}, S_{j}^{y}, S_{j}^{z}\right)$ is calculated using Eq. (6). From $\mathbf{S}_{j}$, the angle $\xi_{j}$ is obtained. The assignment of the value $\xi_{j}$ from $\mathbf{S}_{j}$ is non-trivial since $\xi_{j}$ may be multi-valued. The multi-valuedness of $\xi$ is detected by calculating its winding number.

The winding number of $\xi$ is calculated by using the angular variable $\eta$ in Eq. (7). The difference of $\eta$ between nearest neighbor sites is taken in the range,

$$
-\pi \leq \eta_{\ell}-\eta_{k}<\pi
$$

If $\eta$ is multi-valued, $\xi$ is multi-valued.

The multi-valuedness of $\eta$ is detected if it is rebuilt from the values $\eta_{\ell}-\eta_{k}$. The rebuilding proceeds as follows: first, we obtain the value for $\eta_{1}$ in $-\pi \leq \eta_{1}<\pi$; the step where value of $\eta_{\ell}$ is obtained from the already evaluated values of $\eta_{k}$ is given by

$$
\eta_{\ell}=\eta_{k}+\left(\eta_{\ell}-\eta_{k}\right)
$$


where the sites $\ell$ and $k$ are nearest neighbors connected by a bond, i.e., the line segment that connects the $\ell$ th and $k$ th sites. From $\eta_{j}$, the value of $\xi_{j}$ is obtained using the relation in Eq. (7). This process is continued until values at all accessible sites are evaluated once and only once. If $\eta$ is multi-valued, jump of values of multiple of $2 \pi$ exist across bonds that are not used for the rebuilding.

In the coefficient $\tilde{D}_{j \sigma}^{\gamma}$, the multi-valuedness arising from the phase factors $e^{ \pm i \frac{\xi_{j}}{2}}$ may be hidden if spin-vortices exist. This solution may correspond to the one using the basis in Eq. (11) with $\chi=$ constant, which may violate the condition in Eq. (14). Then, $\{|\tilde{\gamma}\rangle\}$ may not be single-valued.

We can construct the the single-valued basis $\{|\gamma\rangle\}$ from $\{|\tilde{\gamma}\rangle\}$ in the following way: first, we introduce the following new basis to separate the multi-valued phase factors $e^{ \pm i \frac{\xi_{j}}{2}}$;

$$
\begin{gathered}
\tilde{d}_{j \uparrow}=e^{i \frac{\xi_{j}}{2}} c_{j \uparrow}, \\
\tilde{d}_{j \downarrow}=e^{-i \frac{\xi_{j}}{2}} c_{j \downarrow}
\end{gathered}
$$

Then, the Hartree-Fock Hamiltonian becomes

$$
\begin{aligned}
H_{\mathrm{EHFS}}^{H F} & =-t \sum_{\langle i, j\rangle_{1}}\left(e^{\frac{i}{2}\left(\xi_{i}-\xi_{j}\right)} \tilde{d}_{i \uparrow}^{\dagger} \tilde{d}_{j \uparrow}+e^{-\frac{i}{2}\left(\xi_{i}-\xi_{j}\right)} \tilde{d}_{i \downarrow}^{\dagger} \tilde{d}_{j \downarrow}+\text { h.c. }\right) \\
& +U \sum_{j}\left[\left(\frac{n_{j}}{2}-S_{j}^{z}\right) \tilde{d}_{j \uparrow}^{\dagger} \tilde{d}_{j \uparrow}+\left(\frac{n_{j}}{2}+S_{j}^{z}\right) \tilde{d}_{j \downarrow}^{\dagger} \tilde{d}_{j \downarrow}-\left(S_{j}^{x}-i S_{j}^{y}\right) e^{i \xi_{j}} \tilde{d}_{j \uparrow}^{\dagger} \tilde{d}_{j \downarrow}\right. \\
& \left.-\left(S_{j}^{x}+i S_{j}^{y}\right) e^{-i \xi_{j}} \tilde{d}_{j \downarrow}^{\dagger} \tilde{d}_{j \uparrow}\right]
\end{aligned}
$$

By self-consistently diagonalizing the above Hamiltonian, we again obtain the multivalued basis function;

$$
\begin{aligned}
|\tilde{\gamma}\rangle & =\sum_{j}\left[D_{j \uparrow}^{\gamma} \tilde{d}_{j \uparrow}^{\dagger}+D_{j \downarrow}^{\gamma} \tilde{d}_{j \downarrow}^{\dagger}\right]|\mathrm{vac}\rangle \\
& =\sum_{j}\left[e^{-i \frac{\xi_{j}}{2}} D_{j \uparrow}^{\gamma} c_{j \uparrow}^{\dagger}+e^{i \frac{\xi_{j}}{2}} D_{j \downarrow}^{\gamma} c_{j \downarrow}^{\dagger}\right]|\mathrm{vac}\rangle
\end{aligned}
$$

The phase factors $e^{ \pm i \frac{\xi_{j}}{2}}$ are separated, and single-valued coefficients $D_{j \sigma}^{\gamma}$ 's are obtained.

By adding the phase factor introducing $e^{-i \frac{\chi}{2}}$, the single-valued wave functions are constructed as follows,

$$
|\gamma\rangle=\sum_{j} e^{-i \frac{\chi_{j}}{2}}\left[e^{-i \frac{\xi_{j}}{2}} D_{j \uparrow}^{\gamma} c_{j \uparrow}^{\dagger}+e^{i \frac{\xi_{j}}{2}} D_{j \downarrow}^{\gamma} c_{j \downarrow}^{\dagger}\right]|\mathrm{vac}\rangle
$$


where $\chi$ is an angular variable that satisfies the condition in Eq. (14).

Let us explain how $\chi$ is obtained. The angular variable $\chi$ is obtained by the condition that $\nabla \chi$ minimizes the total energy. For this purpose, we employ the following functional,

$$
F[\nabla \chi]=E[\nabla \chi]+\sum_{\ell=1}^{N_{\text {loop }}} \lambda_{\ell}\left(\oint_{C_{\ell}} \nabla \chi \cdot d \mathbf{r}-2 \pi w_{\ell}\right)
$$

where $E[\nabla \chi]$ is the total energy depends on $\nabla \chi$. The second term in the right-hand side is the term arising from the constraints; $\lambda_{\ell}$ is the Lagrange multiplier, $w_{\ell}$ is the winding number of $\chi$ along a loop $C_{\ell}$, and $N_{\text {loop }}$ is the number of independent loops. Note that with $N_{\text {loop }}$ independent loops, any loop in the system can be constructed by the combination of them. A set of values for $w_{\ell}$ specifies a particular current distribution; in other words, by changing the values of $w_{\ell}$ for the independent loops, solutions with different current distributions are obtained.

From the stationary condition of $F[\nabla \chi]$, the optimized $\nabla \chi$ is given as the solution of

$$
0=\frac{\delta F[\nabla \chi]}{\delta \nabla \chi}=\frac{\delta E[\nabla \chi]}{\delta \nabla \chi}+\sum_{\ell=1}^{N_{\text {loop }}} \lambda_{\ell} \frac{\delta}{\delta \nabla \chi} \oint_{C_{\ell}} \nabla \chi \cdot d \mathbf{r}
$$

with the constraints,

$$
\oint_{C_{\ell}} \nabla \chi \cdot d \mathbf{r}-2 \pi w_{\ell}=0 \text { for all independent loops } C_{\ell}
$$

Since the current density is given in general by

$$
\mathbf{j}=-c \frac{\delta E}{\delta \mathbf{A}^{\mathrm{em}}}
$$

where $\mathbf{A}^{\mathrm{em}}$ is the electromagnetic vector potential, and adding the phase factor $e^{-i \frac{\chi_{j}}{2}}$ in Eq. (11) corresponds to introducing the vector potential $\mathbf{A}^{\text {fic }}$ in Eq. (12), the current density is expressed as

$$
\mathbf{j}=-c \frac{\delta E}{\delta \mathbf{A}^{\text {fic }}}=-\frac{2 q}{\hbar} \frac{\delta E}{\delta \nabla \chi}
$$

Then, from Eq. (25), the current density is given by

$$
\mathbf{j}=\frac{2 q}{\hbar} \sum_{\ell=1}^{N_{\text {loop }}} \lambda_{\ell} \frac{\delta}{\delta \nabla \chi} \oint_{C_{\ell}} \nabla \chi \cdot d \mathbf{r}
$$

This is the current density formula for the SVILCs. This is a stable current protected by the topological integers, winding numbers. It will, however, fluctuate at temperatures above 
$T_{\mathrm{c}}$ since each loop current has the freedom in the direction of the flow, and this fluctuation will explain the fluctuation of the vortex current of the coherence length size appeared in the estimate of $T_{\mathrm{c}}$ by [16]. If the constraint is absent $\left(\lambda_{\ell}=0\right.$ for all $\left.\ell\right)$, the current is zero, indicating that it arises due to the single-valued requirement of the wave function.

As a function of $\nabla \chi$, the expectation value of the hopping term is calculated as

$$
K[\nabla \chi]=-t \sum_{\langle k, j\rangle_{1}} \sum_{\gamma=1}^{N}\left(e^{\frac{i}{2}\left(\chi_{k}-\chi_{j}\right)} e^{\frac{i}{2}\left(\xi_{k}-\xi_{j}\right)}\left(D_{k \uparrow}^{\gamma}\right)^{*} D_{j \uparrow}^{\gamma}+e^{\frac{i}{2}\left(\chi_{k}-\chi_{j}\right)} e^{-\frac{i}{2}\left(\xi_{k}-\xi_{j}\right)}\left(\left(D_{k \downarrow}^{\gamma}\right)^{*} D_{j \downarrow}^{\gamma}\right)+\right.\text { c.c. }
$$

where the sum is taken over occupied orbitals $|\gamma\rangle$.

Using the relation, $\frac{\delta E[\nabla \chi]}{\delta \nabla \chi}=\frac{\delta K[\nabla \chi]}{\delta \nabla \chi}$, the following equations for $\nabla \chi$ and $\lambda_{\ell}$ are obtained from Eq. (25);

$$
\frac{\delta K[\nabla \chi]}{\delta \nabla \chi}+\sum_{\ell=1}^{N_{\text {loop }}} \lambda_{\ell} \frac{\delta}{\delta \nabla \chi(\mathbf{x})} \oint_{C_{\ell}} \nabla \chi \cdot d \mathbf{r}=0
$$

In the discrete lattice, Eqs. (31) and (26) become,

$$
\begin{aligned}
& \frac{\partial K}{\partial \tau_{k \leftarrow j}}+\sum_{\ell=1}^{N_{\text {loop }}} \lambda_{\ell} \frac{\partial}{\partial \tau_{k \leftarrow j}} \sum_{k \leftarrow j} \tau_{k \leftarrow j} L_{k \leftarrow j}^{\ell}=0 \\
& \sum_{k \leftarrow j} \tau_{k \leftarrow j} L_{k \leftarrow j}^{\ell}=2 \pi w_{\ell}
\end{aligned}
$$

where $L_{k \leftarrow j}^{\ell}$ is defined by

$$
L_{k \leftarrow j}^{\ell}= \begin{cases}-1 & \text { if } \tau_{k \leftarrow j} \text { is in } C_{\ell} \text { with clockwise direction } \\ 1 & \text { if } \tau_{k \leftarrow j} \text { is in } C_{\ell} \text { with counterclockwise direction } \\ 0 & \text { if } \tau_{k \leftarrow j} \text { is not in } C_{\ell}\end{cases}
$$

and $\tau_{k \leftarrow j}$ denotes the phase difference over bonds

$$
\tau_{k \leftarrow j}=\chi_{k}-\chi_{j}
$$

We take the branch of $\chi$ so that the difference of $\chi$ between nearby sites is in the range,

$$
-\pi \leq \chi_{\ell}-\chi_{k}<\pi
$$

The equation (32) is calculated as

$$
t \sin \frac{\tau_{k \leftarrow j}}{2} F_{k \leftarrow j}+\sum_{\ell} L_{k \leftarrow j}^{\ell} \lambda_{\ell}=0
$$


where

$$
F_{k \leftarrow j}=\sum_{\gamma=1}^{N}\left[e^{\frac{i}{2}\left(\xi_{k}-\xi_{i}\right)}\left(D_{k \uparrow}^{\gamma}\right)^{*} D_{j \uparrow}^{\gamma}+e^{-\frac{i}{2}\left(\xi_{k}-\xi_{i}\right)}\left(D_{k \downarrow}^{\gamma}\right)^{*} D_{j \downarrow}^{\gamma}\right]
$$

We must solve the system of equations composed of Eq. (37) and Eq. (33) for $\tau_{k \leftarrow j}$ and $\lambda_{\ell}$. After obtaining $\tau_{k \leftarrow j}$ and $\lambda_{\ell}$, the current is calculated as

$$
J_{k \leftarrow j}=\frac{2 q}{\hbar} \sum_{\ell} L_{k \leftarrow j}^{\ell} \lambda_{\ell}
$$

from Eq. (29).

Values of $\tau_{k \leftarrow j}$ are calculated in the following way; first we split $\tau_{k \leftarrow j}$ as

$$
\tau_{k \leftarrow j}=\tau_{k \leftarrow j}^{0}+\delta \tau_{k \leftarrow j}
$$

where $\tau_{k \leftarrow j}^{0}$ is obtained by linearizing Eq. (37) as

$$
\tau_{k \leftarrow j}^{0} \frac{t}{2} F_{k \leftarrow j}+\sum_{\ell} L_{k \leftarrow j}^{\ell} \lambda_{\ell}=0
$$

Then, using Eqs. (33) and (41), we obtain the following system of linear equations;

$$
\sum_{\ell^{\prime}} M_{\ell}^{\ell^{\prime}} \lambda_{\ell^{\prime}}=2 \pi w_{\ell}
$$

where

$$
\begin{aligned}
M_{\ell}^{\ell^{\prime}} & =-\sum_{k \leftarrow j} \frac{2}{t F_{k \leftarrow j}} L_{k \leftarrow j}^{\ell} L_{k \leftarrow j}^{\ell^{\prime}} \\
& =\left\{\begin{array}{cc}
\sum_{k \leftarrow j \in C_{\ell} \cap C_{\ell^{\prime}}} 2\left(t F_{k \leftarrow j}\right)^{-1} & \ell \neq \ell^{\prime} \\
-\sum_{k \leftarrow j \in C_{\ell}} 2\left(t F_{k \leftarrow j}\right)^{-1} & \ell=\ell^{\prime}
\end{array}\right.
\end{aligned}
$$

$\lambda_{\ell}$ 's are obtained from the equation in Eq. (42). After the calculation of $\lambda_{\ell}$ 's, $\tau_{k \leftarrow j}^{0}$ 's are obtained from Eq. (41).

Next, we evaluate $\delta \tau_{k \leftarrow j}$; assuming that $\delta \tau_{k \leftarrow j}$ is very small, Eq. (37) is written as

$$
2 \sin \frac{\tau_{k \leftarrow j}^{0}}{2}+\delta \tau_{k \leftarrow j} \cos \frac{\tau_{k \leftarrow j}^{0}}{2}+2\left(t F_{k \leftarrow j}\right)^{-1} \sum_{\ell} L_{k \leftarrow j}^{\ell} \lambda_{\ell}=0
$$

The winding numbers calculated with $\delta \tau_{k \leftarrow j}$ 's are zero; thus, Eq. (33) becomes

$$
\sum_{k \leftarrow j} L_{k \leftarrow j}^{\ell} \delta \tau_{k \leftarrow j}=0
$$


Using Eqs. (44) and (45), we have

$$
\sum_{\ell^{\prime}} N_{\ell}^{\ell^{\prime}} \lambda_{\ell^{\prime}}=-\sum_{k \leftarrow j \in C_{\ell}} L_{k \leftarrow j}^{\ell} \tan \frac{\tau_{k \leftarrow j}^{0}}{2}
$$

for the equation for $\lambda_{\ell}$, where

$$
\begin{aligned}
N_{\ell}^{\ell^{\prime}} & =\sum_{k \leftarrow j}\left(t F_{k \leftarrow j}\right)^{-1} \cos ^{-1} \frac{\tau_{k \leftarrow j}^{0}}{2} L_{k \leftarrow j}^{\ell} L_{k \leftarrow j}^{\ell^{\prime}} \\
& =\left\{\begin{array}{cc}
-\sum_{k \leftarrow j \in C_{\ell} \cap C_{\ell^{\prime}}}\left(t F_{k \leftarrow j} \cos \frac{\tau_{k \leftarrow j}^{0}}{2}\right)^{-1} & \ell \neq \ell^{\prime} \\
\sum_{k \leftarrow j \in C_{\ell}}\left(t F_{k \leftarrow j} \cos \frac{\tau_{k \leftarrow j}^{0}}{2}\right)^{-1} & \ell=\ell^{\prime}
\end{array}\right.
\end{aligned}
$$

After obtaining $\lambda_{\ell}$ 's from Eq. (46), $\delta \tau_{k \leftarrow j}$ 's are calculated using Eq. (44). Next, after updating $\tau_{k \leftarrow j}^{0}$ as the sum of the previous $\tau_{k \leftarrow j}^{0}$ and the obtained $\delta \tau_{k \leftarrow j}$, we solve Eq. (46); then, new $\delta \tau_{k \leftarrow j}$ 's are calculated again using Eq. (44); we repeat this cycle till $\delta \tau_{k \leftarrow j}$ becomes sufficiently small.

The calculation with the current feeding boundary condition is also possible [7]. In this case, Eq. (32) is modified as

$$
\frac{\partial K}{\partial \tau_{k \leftarrow j}}+\sum_{\ell=1}^{N_{\text {loop }}} \lambda_{\ell} \frac{\partial}{\partial \tau_{k \leftarrow j}} \sum_{k \leftarrow j} \tau_{k \leftarrow j} L_{k \leftarrow j}^{\ell}+\sum_{\ell=1}^{N_{\text {loop }}^{\text {ext }}} \lambda_{\ell}^{\text {ext }} \frac{\partial}{\partial \tau_{k \leftarrow j}} \sum_{k \leftarrow j} \tau_{k \leftarrow j} L_{k \leftarrow j}^{\ell}=0
$$

where the sum over $N_{\text {loop }}^{\text {ext }}$ external loops are included; each external loop is a loop that includes a bond directly connecting two current feeding sites on the edges; this bond is not a part of the lattice, but the rest of the bonds in the external loop are those of the lattice. The resulting current is independent of the choice of the bonds in the lattice.

Using Eq. (39), $\lambda_{\ell}^{\text {ext }}$ is related to the feeding current $J_{k \leftarrow j}^{\text {ext }}$ as

$$
J_{k \leftarrow j}^{\mathrm{ext}}=\frac{2 q}{\hbar} \sum_{\ell} L_{k \leftarrow j}^{\ell} \lambda_{\ell}^{\mathrm{ext}}
$$

This will conserve the charge for the current feeding boundary condition where the current flow-in at the $k$ th site and flow-out from the $j$ th site. This boundary condition may be viewed as the situation where current flows in from the lead connected to the $k$ th site, and flows out to the lead connected to the $j$ th site. The equation (49) is solved for $\lambda_{\ell}^{\text {ext }}$, and the obtained $\lambda_{\ell}^{\text {ext's }}$ are substituted in Eq. (48).

For the construction of basis $\{|\gamma\rangle\}$, values of $\eta$ and $\chi$ at each site have to be rebuilt from the values $\eta_{\ell}-\eta_{k}$ and $\chi_{\ell}-\chi_{k}$, respectively. The rebuilding of $\chi$ can be done in the similar 
manner as that of $\xi$. We have $\tau_{k \leftarrow j}=\chi_{k}-\chi_{j}$ for all the bonds, thus, we rebuild $\chi$ from them. First, we pick a value for the initial $\chi_{1}$ ( say $\chi_{1}=0$ ); the step where value $\chi_{\ell}$ is derived from the already evaluated value of $\chi_{k}$ is given by

$$
\chi_{\ell}=\chi_{k}+\left(\chi_{\ell}-\chi_{k}\right)
$$

where the sites $\ell$ and $k$ are connected by a bond in the path. This process is continued until values at all accessible sites are evaluated once and only once. Using $\xi_{j}$ and $\chi_{j}$ obtained from the rebuilding, we obtain values of $\xi_{j}-\chi_{j}$ and $\xi_{j}+\chi_{j}$.

Since the values of $\xi$ and $\chi$ are path-dependent they have $2 \pi n$ ( $n$ is an integer) jumps between sites that are connected by bonds but not used during the rebuilding process described above. Due to the condition in Eq. (14), the phase jumps for $\xi \pm \chi$ are $4 \pi n$ ( $n$ is an integer). Then, values of $e^{-i \frac{\xi_{j}+\chi_{j}}{2}}$ and $e^{i \frac{\xi_{j}-\chi_{j}}{2}}$ are path-independent because the $4 \pi n$ jumps are absorbed by $e^{i 2 \pi n}=1$. In this way, the single-valued wave functions $\{|\gamma\rangle\}$ are obtained.

Using occupied levels from $\{|\gamma\rangle\}$, we can construct the Hartree-Fock total electronic wave function. It has the following form

$$
\Phi\left(\mathbf{r}^{(1)}, \cdots, \mathbf{r}^{(N)}\right)=\Phi_{0}\left(\mathbf{r}^{(1)}, \cdots, \mathbf{r}^{(N)}\right) e^{-\frac{i}{2} \sum_{\alpha=1}^{N} \chi\left(\mathbf{r}^{(\alpha)}\right)}
$$

where $\mathbf{r}^{(j)}$ is the coordinate of the $j$ th electron. The phase factor $e^{-\frac{i}{2} \sum_{\alpha=1}^{N} \chi\left(\mathbf{r}^{(\alpha)}\right)}$ arises due to the factor $e^{-i \frac{\chi_{j}}{2}}$ added in Eq. (23). $\Phi_{0}$ is a multi-valued currentless wave function; $e^{-\frac{i}{2} \sum_{\alpha=1}^{N} \chi\left(\mathbf{r}^{(\alpha)}\right)}$ makes the total wave function single-valued by compensating the multivaluedness of $\Phi_{0}$, and generates the supercurrent.

\section{RESULTS}

Now we present numerical results for supercurrent carrying states obtained by the method described in the previous section. The parameters used are $U=8 t[22]$ and $J^{\prime}=0.5 J$ or $J^{\prime}=0$, where $J=4 t^{2} / U$. We found that for the self-consistent solutions with the spinvortices given below, the resulting $\zeta$ is turned out to be always $\pi / 2$; this means that the spins are lying in the $x-y$ plane when the spin-vortices are formed in the present model. The contribution from Eq. (3) is included as its expectation value calculated using the Hartree-Fock single determinant wave function.

The SVQ in the $\mathrm{CuO}_{2}$ plane of the cuprate embedded in the antiferromagnetic background is depicted in Fig. 1b. In Fig. 1c-f, four current patterns arising from the single SVQ spin- 
a

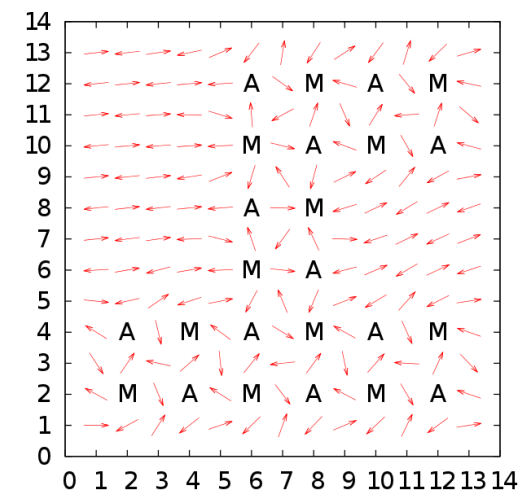

C

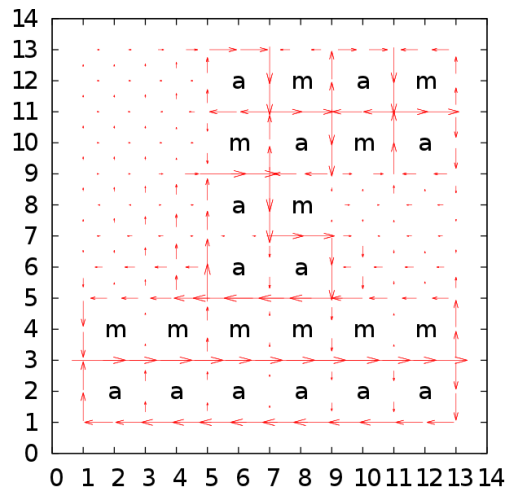

b

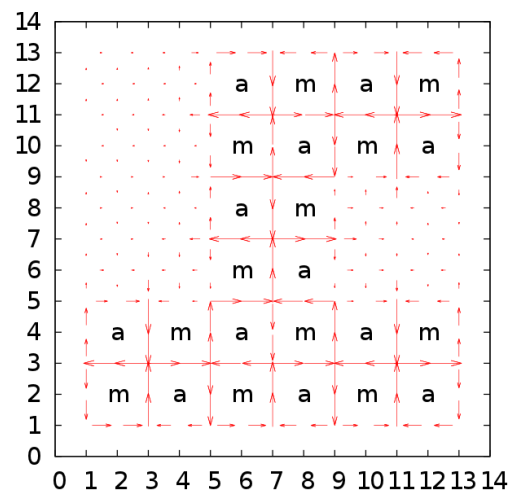

d

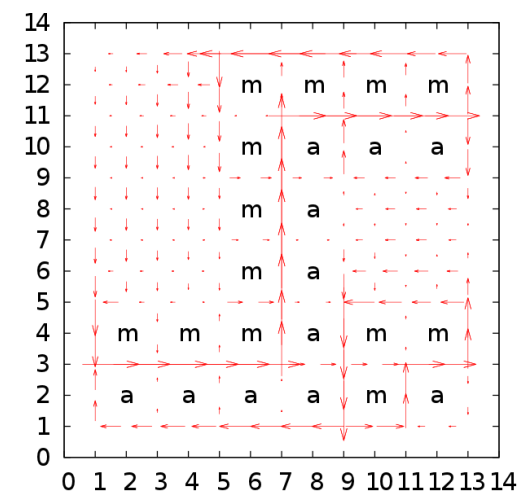

e

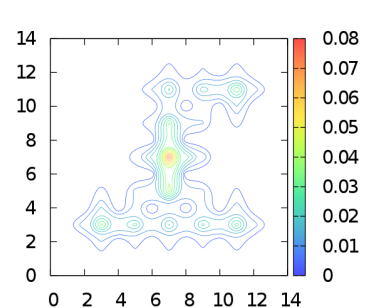

$\mathrm{h}$

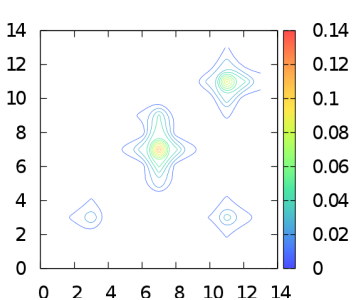

$\mathrm{f}$

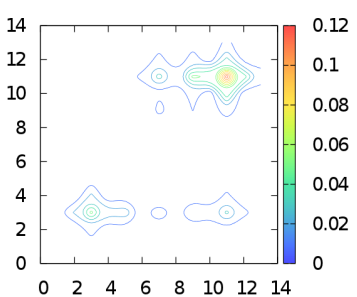

i

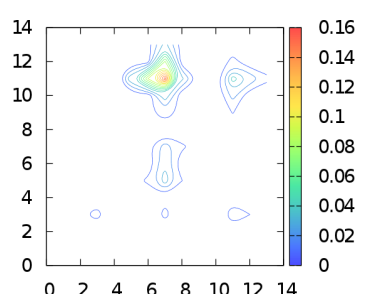

g

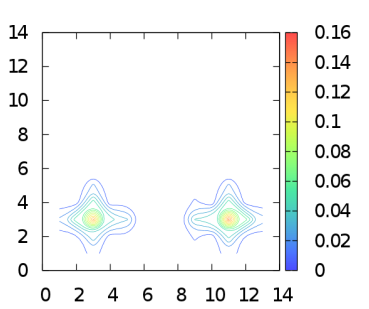

j

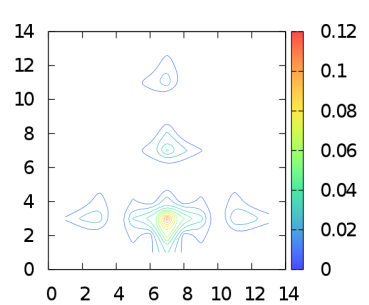

FIG. 2: Plots of the spin configuration, current pattern, and density map of the single particle orbital. a, Spin configuration. b, The minimal energy current distribution. c, Current distribution with flow from $(1,3)$ to $(13,3)$. This state is higher in energy than b by $2.03 t$. d, Current distribution with flow from $(1,3)$ via $(7,3)$, and $(7,11)$ to $(13,11)$. This state is higher in energy than $\mathbf{b}$ by $4.17 t$. $\mathbf{e - j}$, Density maps of the probability amplitude for first to sixth highest energy occupied single particle orbitals. For the current in $\mathbf{b}$, the energy decreasing order of the orbitals is $\mathbf{e}-\mathbf{j}$; for $\mathbf{c}$, $\mathbf{e}, \mathbf{f}, \mathbf{h}, \mathbf{i}, \mathbf{g}$, and $\mathbf{j}$; for $\mathbf{d}, \mathbf{f}, \mathbf{g}, \mathbf{i}, \mathbf{e}, \mathbf{j}$, and $\mathbf{h}$. 
texture in Fig. 1b are shown. Different current patterns are obtained by choosing different values of $w_{\ell}$ in Eq. (24); 'm' and 'a' indicate the centers of the SVILCs with $w_{\ell}=1$ and $w_{\ell}=-1$, respectively. The total energy of the single SVQ system in Fig. $1 \mathbf{b}$ is higher than that of the antiferromagnetic case in Fig. 1a. The SVQ state becomes the ground state if we include the superexchange interaction given in Eq. (3) with sufficiently large $J^{\prime}$. With the value $J^{\prime}=0.5 J$, where $J=4 t^{2} / U$, the state with the spin-vortices becomes more stable than the AF case by $0.21 t$. The lowest energy state is the one with the current pattern in Fig. 1c. The current is practically zero for this minimal energy current pattern. A horizontal current arises between rows of ' $a$ ' and ' $\mathrm{m}$ ' in the current pattern in Fig. 1d; this state is degenerate with the one with a vertical current in Fig. 1e. The state with a large current in Fig. 1f has a significantly larger energy than the other current pattern state.

The self-consistent numerical calculations indicate that the SVQ is a stable unit. As the hole doping is increased, the $\mathrm{CuO}_{2}$ plane will be covered by them. In Fig. 2a, a spinconfiguration with six SVQs is shown. The cluster of the localized holes observed in the experiment [18] may be a collection of the SVQs such as this.

The current by the SVILCs flows through the channel generated by the SVQs. Energetically favorable current patterns are those constructed with the horizontal current unit depicted in Fig. 1d and its degenerate partner with the vertical current in Fig. 1e. Two examples are shown in Fig. 2c-d. They are stable since the SVILCs are protected by their topological integers. Due to this stability, they may be regarded as the local supercurrent. When the network of the spin-vortices is extended and covers the whole $\mathrm{CuO}_{2}$ plane, the macroscopic superconductivity will be realized.

As shown in the density maps of the probability amplitude of single-particle orbitals in Fig. 2e-j, the states just below the Fermi energy have large probability density at the centers of the SVQs. When electrons in those states are extracted, the SVQ will be destroyed. This means that the extraction of the electrons near the Fermi level costs an extra energy. This may explain the energy gap observed in the STM.

The results for the current feeding boundary condition are depicted in Fig. 3. This shows that the present supercurrent transfers electric charge that enters into one end and exits from another without a voltage difference. 
a

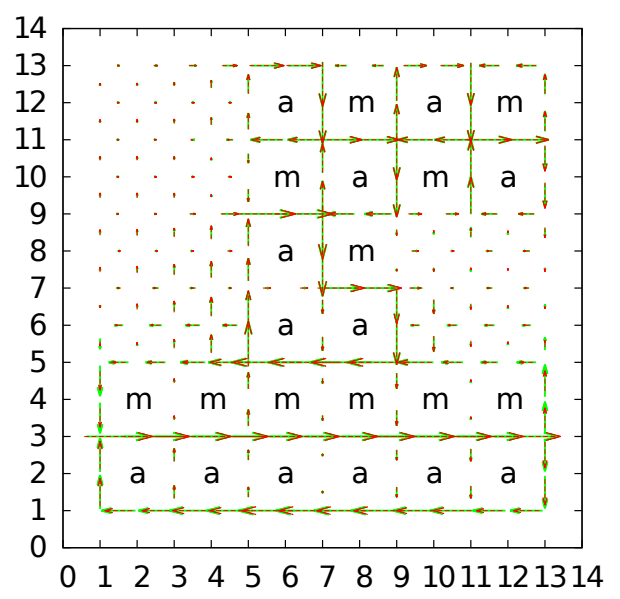

b

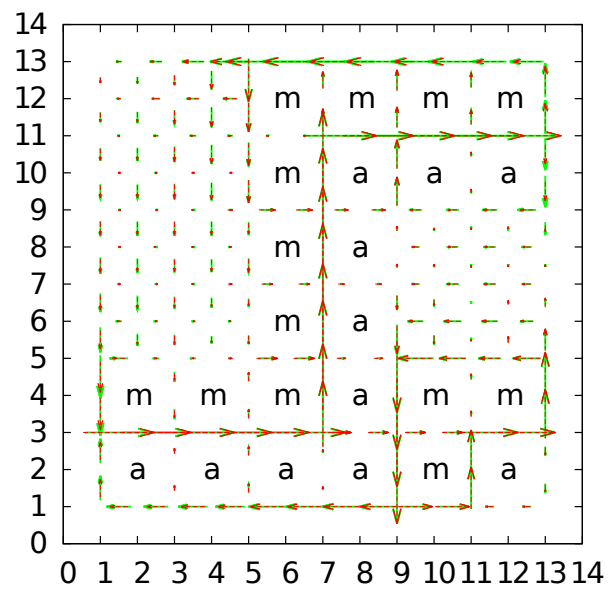

FIG. 3: Plots of current flows for the channel made by the spin configuration in Fig. 2a. The current flow with current feeding boundary condition (red) is superimposed on the closed current pattern (green). a, the current of 0.1 et/ $\hbar$ flow-in at $(1,3)$ and flow-out at $(13,3)$. $\mathbf{b}$, the current of 0.1 et $/ \hbar$ flow-in at $(1,3)$ and flow-out at $(13,11)$.

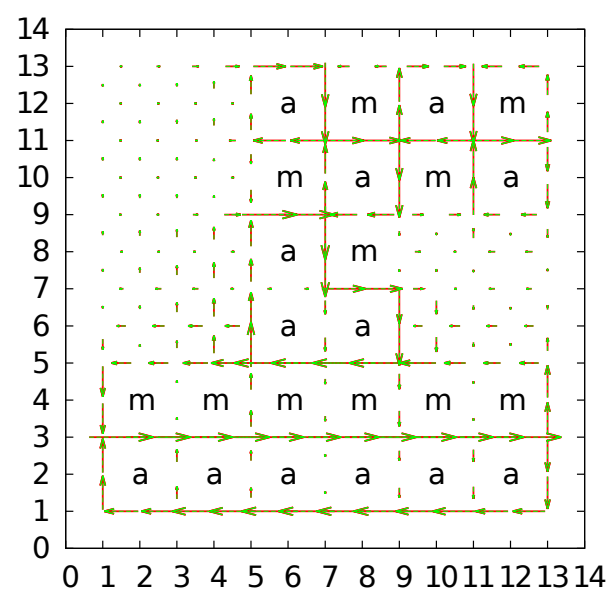

FIG. 4: Plots of current flow in Fig. 2a. The current flow calculated with Eq. (39)(green) is superimposed on that calculated by Eq. (52) (red).

The current can be also calculated as the following expectation value

$$
J_{k \leftarrow j}^{\mathrm{wave}}=i \frac{q t}{\hbar}\left\langle\sum_{\sigma} c_{k \sigma}^{\dagger} c_{j \sigma}-c_{j \sigma}^{\dagger} c_{k \sigma}\right\rangle
$$

using the total wave function $\Phi\left(\mathbf{r}^{(1)}, \cdots, \mathbf{r}^{(N)}\right)$ in Eq. (51). In Fig. (4), the result is compared with the one calculated using Eq. (39), showing that the two results are identical as they 
should be. The current is generated by the phase factor $e^{-\frac{i}{2} \sum_{\alpha=1}^{N} \chi\left(\mathbf{r}^{(\alpha)}\right)}$.

\section{GINZBURG-LANDAU EQUATION OF THE SPIN-VORTEX MODEL: DERIVATION OF THE FLUX QUANTUM $h c / 2 e$ BY THE SUPERCURRENT OF SVILCS}

In this section, we derive the equation corresponding to the Ginzburg-Landau equation using the wave function in Eq. (51). It is also shown that the flux quantum $h c / 2 e$ is obtained for the supercurrent generated by the SVILCs.

In the following derivation, we assume that $\Phi_{0}$ part of the wave function $\Phi$ in Eq. (51) is rigid in the sense that it is the ground state characterized only by the electron density $\rho$ calculated from $\Phi_{0}$. We further assume that $\rho$ is time-independent; the time dependence only exists in $\chi$. Then, by treating $\chi$ and $\rho$ as collective modes for the superconductivity, we will show that the macroscopic equation corresponding to the Ginzburg-Landau equation at $T=0 \mathrm{~K}$ is obtained.

Let us consider the following energy functional

$$
\begin{aligned}
E\left[\Phi, \mathbf{A}^{\mathrm{em}}\right] & =\left\langle\Phi\left|\left\{\sum_{\alpha=1}^{\mathrm{N}} \frac{1}{2 m}\left[\mathbf{p}^{(\alpha)}-\frac{q}{c} \mathbf{A}^{\mathrm{em}}\left(\mathbf{r}^{(\alpha)}\right)\right]^{2}+H_{\mathrm{int}}\right\}\right| \Phi\right\rangle \\
& +\frac{1}{8 \pi} \int d^{3} r\left(\nabla \times \mathbf{A}^{\mathrm{em}}\right)^{2}
\end{aligned}
$$

where $m$ is the effective mass of the electron, $q=-e$ is the charge of the electron, $H_{\text {int }}$ is the Hamiltonian for interactions, $\mathbf{A}^{\mathrm{em}}$ is the vector potential for electromagnetic field, and the last term is the magnetic field energy. Actually, due to the large anisotropy, the effective mass in the $a b$ plane and $c$ direction are different in the cuprate; however, for simplicity, we use the same effective mass in the following.

By using the fact that the current density for $\Phi_{0}$ is zero, $E\left[\Phi, \mathbf{A}^{\mathrm{em}}\right]$ is expressed with $\rho$ and $\chi$ as

$$
\begin{aligned}
E\left[\rho, \chi, \mathbf{A}^{\mathrm{em}}\right] & =E_{0}[\rho]+\frac{1}{2 m} \int d^{3} r \rho\left(\frac{\hbar}{2} \nabla \chi+\frac{q}{c} \mathbf{A}^{\mathrm{em}}\right)^{2} \\
& +\frac{1}{8 \pi} \int d^{3} r\left(\nabla \times \mathbf{A}^{\mathrm{em}}\right)^{2}
\end{aligned}
$$


where $E_{0}[\rho]$ is the ground state energy functional for the electron density $\rho$ given by

$$
E_{0}[\rho]=\left\langle\Phi_{0}\left|\left\{\sum_{\alpha=1}^{\mathrm{N}} \frac{1}{2 m}\left(\mathbf{p}^{(\alpha)}\right)^{2}+H_{\mathrm{int}}\right\}\right| \Phi_{0}\right\rangle
$$

We regard $E\left[\rho, \chi, \mathbf{A}^{\mathrm{em}}\right]$ as the functional to be minimized to obtain the stable state. The stationary condition of $E\left[\rho, \chi, \mathbf{A}^{\mathrm{em}}\right]$ with respect to the variation of $\mathbf{A}^{\mathrm{em}}$ yields,

$$
\nabla \times \mathbf{B}^{\mathrm{em}}=\frac{4 \pi q \rho}{m c}\left(\frac{\hbar}{2} \nabla \chi+\frac{q}{c} \mathbf{A}^{\mathrm{em}}\right)
$$

where $\mathbf{B}^{\mathrm{em}}=\nabla \times \mathbf{A}^{\mathrm{em}}$. This is one of Maxwell's equations. It indicates that the electric current density is given by

$$
\mathbf{j}=\frac{q \rho}{m}\left(\frac{\hbar}{2} \nabla \chi+\frac{q}{c} \mathbf{A}^{\mathrm{em}}\right)
$$

This is the London equation [23].

The stationary condition of $E\left[\rho, \chi, \mathbf{A}^{\mathrm{em}}\right]$ with respect to the variation of $\chi$ yields,

$$
\nabla \cdot \mathbf{j}=0
$$

This shows that the optimized $\chi$ ensures the conservation of electric charge.

The equation (57) indicates that the appearance of $\chi$ gives rise to the vector potential $\mathbf{A}^{\text {fic }}$ given in Eq. (12), and the effective vector potential in the system becomes the following sum

$$
\mathbf{A}^{\mathrm{eff}}=\mathbf{A}^{\mathrm{fic}}+\mathbf{A}^{\mathrm{em}}
$$

With the optimized $\nabla \chi$ (equivalent to the optimized $\mathbf{A}^{\text {fic }}$ ), $\mathbf{A}^{\text {eff }}$ is gauge invariant: if we employ a different vector potential of electromagnetism $\mathbf{A}^{\prime e m}=\mathbf{A}^{\mathrm{em}}+\nabla f$, where $f$ is a singlevalued function, the optimized fictitious vector potential is modified as $\mathbf{A}^{\prime \text { fic }}=\mathbf{A}^{\text {fic }}-\nabla f$; thus, the effective vector potential $\mathbf{A}^{\mathrm{eff}}$ remains the same.

From Eqs. (56) and (57), the Meissner effect is explained. This means that the current density is zero in the bulk. Actually, the current density in this case should be regarded as an average over a coarse-grained region since microscopic loop currents given in Eq. (29), such as shown in Fig. 1c, exist.

Let us consider a ring-shaped system and take a loop $C$ that goes around the hole of the ring through the bulk region where $\mathbf{j}=0$ in the above-mentioned sense. Then, from Eq. (57), we have

$$
0=\oint_{C}\left(\frac{\hbar}{2} \nabla \chi+\frac{q}{c} \mathbf{A}^{\mathrm{em}}\right) \cdot d \mathbf{r}
$$


Thus, the flux through the loop $C$ is given by

$$
\oint_{C} \mathbf{A}^{\mathrm{em}} \cdot d \mathbf{r}=-\frac{c \hbar}{2 q} \oint_{C} \nabla \chi \cdot d \mathbf{r}=\frac{c \hbar}{2 e} \oint_{C} \nabla \chi \cdot d \mathbf{r}=\frac{c h}{2 e} w_{C}
$$

where $w_{C}$ is the winding number of $\chi$ for the loop $C$. This shows that the magnetic flux is a multiple of $\frac{c \hbar}{2 e}$. Usually, $2 e$ in the flux quantum is regarded as the evidence that the charge carriers in superconductors are paired electrons. However, it is obtained without assuming the presence of electron pairs here.

Let us show that the variables $\rho$ and $\chi$ are canonical conjugate; for that purpose, we consider the following Lagrangian

$$
L=\left\langle\Phi\left|i \hbar \frac{\partial}{\partial t}-\left\{\sum_{\alpha=1}^{\mathrm{N}} \frac{1}{2 m}\left[\mathbf{p}^{(\alpha)}-\frac{q}{c} \mathbf{A}^{\mathrm{em}}\left(\mathbf{r}^{(\alpha)}\right)\right]^{2}+H_{\mathrm{int}}\right\}\right| \Phi\right\rangle
$$

We only consider the case where $\chi$ is time-dependent but $\rho$ is time-independent. Then, the Lagrangian is calculated as

$$
L=\int d^{3} r \hbar \frac{\dot{\chi}}{2} \rho-E_{0}[\rho]-\frac{1}{2 m} \int d^{3} r \rho\left(\frac{\hbar}{2} \nabla \chi+\frac{q}{c} \mathbf{A}^{\mathrm{em}}\right)^{2}
$$

where Eq. (54) is used.

From Eq. (63), the canonical conjugate variable to $\chi, p_{\chi}$, is obtained as

$$
p_{\chi}=\frac{\delta L}{\delta \dot{\chi}}=\frac{\hbar}{2} \rho
$$

The Hamiltonian is constructed from $L$ as

$$
H=E_{0}[\rho]+\frac{1}{2 m} \int d^{3} r \rho\left(\frac{\hbar}{2} \nabla \chi+\frac{q}{c} \mathbf{A}^{\mathrm{em}}\right)^{2}
$$

Then, the Hamiltonian equations are given by

$$
\begin{aligned}
& \dot{\rho}=-\frac{2}{\hbar} \frac{\delta H}{\delta \chi}=0 \\
& \dot{\chi}=\frac{2}{\hbar} \frac{\delta H}{\delta \rho}
\end{aligned}
$$

Since $\rho$ is assumed to be time-independent, the condition in Eq. (66) is obtained. It is shown to yield the conservation of the charge given in Eq. (58) [5].

The commutation relation Eq. (64) and Hamilton's equations Eqs. (66) and (67) indicate that the present superconducting state may be regarded as a coherent state with the 
eigenvalue $(\rho / 2)^{1 / 2} e^{-i \chi}$ for the operator $(\hat{\rho} / 2)^{1 / 2} e^{-i \hat{\chi}}$, where $\hat{\rho}$ and $\hat{\chi}$ satisfy the following commutation relation,

$$
\left[\hat{\chi}(\mathbf{r}), \frac{1}{2} \hat{\rho}\left(\mathbf{r}^{\prime}\right)\right]=i \delta^{3}\left(\mathbf{r}-\mathbf{r}^{\prime}\right)
$$

The energy functional in Eq. (54) can be regarded as the one for the single-valued macroscopic wave function $\Psi_{G L}$, given by

$$
\Psi_{G L}=(\rho / 2)^{1 / 2} e^{-i \chi}
$$

where Eq. (54) is expressed as

$$
\begin{aligned}
E\left[\rho, \chi, \mathbf{A}^{\mathrm{em}}\right] & =\frac{1}{4 m} \int d^{3} r\left|\left(\frac{\hbar}{i} \nabla-\frac{2 q}{c} \mathbf{A}^{\mathrm{em}}\right) \Psi_{G L}\right|^{2}+G_{0}\left[2\left|\Psi_{G L}\right|^{2}\right] \\
& +\frac{1}{8 \pi} \int d^{3} r\left(\nabla \times \mathbf{A}^{\mathrm{em}}\right)^{2}
\end{aligned}
$$

with

$$
G_{0}[\rho]=E_{0}[\rho]-\int d^{3} r \frac{\hbar^{2}}{8 m} \frac{(\nabla \rho)^{2}}{\rho}
$$

This functional becomes the one corresponding to the Ginzburg-Landau functional if the quadratic polynomial of $\rho$ is used for $G_{0}[\rho]$. The original Ginzburg-Landau functional is a free energy functional. However, the one given above is an energy functional, thus, it is only valid at $T=0 \mathrm{~K}$.

A Schrödinger equation like equation is obtained from the functional in Eq. (70) with the stationary condition for the variation with respect to $\Psi_{G L}^{*} . \Psi_{G L}$ should be solved as a single-valued function of the coordinate. The mass and charge of the Hamiltonian for $\Psi_{G L}$ are $2 m$ and $2 q$, respectively. The obtained equation is the one for particles with mass $2 m$ and charge $2 q=-2 e . \Psi_{G L}$ is single-valued just like the wave function for the Schrödinger equation.

\section{CONCLUSION}

The supercurrent generation in the cuprate superconductor will be markedly different from that of the BCS superconductor. The spin-vortex model, combined with the atomicscale STM results suggests that the unit of the superconducting state in the cuprate is the 
SVQ of width $4 a$. We expect they will appear in the pseudogap phase and make a network channel of the supercurrent flow. The supercurrent will be a collection of the SVILCs that flows through this network.

The total electronic wave function for the superconducting state is given by $\Phi\left(\mathbf{r}^{(1)}, \cdots, \mathbf{r}^{(N)}\right)=\Phi_{0}\left(\mathbf{r}^{(1)}, \cdots, \mathbf{r}^{(N)}\right) e^{-\frac{i}{2} \sum_{\alpha=1}^{N} \chi\left(\mathbf{r}^{(\alpha)}\right)}$, where $\Phi_{0}$ is a multi-valued currentless wave function and $e^{-\frac{i}{2} \sum_{\alpha=1}^{N} \chi\left(\mathbf{r}^{(\alpha)}\right)}$ is the phase factor that makes $\Phi$ single-valued with respect to the electron coordinates. The latter generates the forced whole system motion imposed by the single-valued requirement of the wave function and generates the supercurrent. The stable current flow that enters from one lead connected to the superconductor and exits into another lead is realized by this whole system motion without a voltage difference between the two leads; this corresponds to the supercurrent observed in the experiment.

We have also obtained the Ginzburg-Landau type equation using the above wave function $\Phi$, and shown that the flux quantum is $c h / 2 e$.

In order to verify the present new mechanism, the detection of the SVILCs is required. For that purpose, we propose to measure the change of the local magnetic field by an irradiation of strong electromagnetic field on a $4 a$ width cluster of holes. If the irradiation causes the transition that alters the current direction of the SVILCs, the change of the magnetic field occurs. Our estimates for the single SVQ case using $t=130 \mathrm{meV}$ are as follows: the transition energy from the ground (Fig. 1c) to the degenerate first excited state (Fig. 1d, e) is about $57 \mathrm{meV}$, and that to the second excited state (Fig. 1f) is about $850 \mathrm{meV}$; the change of the local magnetic field by the transition to the second excited state is about $0.015 \mathrm{~T}$ at $1.2 \mathrm{~nm}$ above the center of the SVQ . In reality, the cluster of holes is composed of many SVQs, thus, real values will be significantly different from the estimates. The details for the alteration of the current direction of the SVILCs will be examined in our future work. A possibility to use SVILCs as qubits of quantum computers will be also discussed.

[1] J. G. Bednorz and K. A. Müller, Z. Phys. B64, 189 (1986).

[2] J. M. Tranquada, H. Woo, T. G. Perring, H. Goka, G. D. Gu, G. Xu, M. Fujita, and K. Yamada, Nature 429, 534 (2004).

[3] S. A. Kivelson, I. P. Bindloss, E. Fradkin, V. Oganesyan, J. M. Tranquada, A. Kapitulnik, 
and C. Howald, Rev. Mod. Phys. 75, 1201 (2003).

[4] H. Koizumi, J. Phys. Soc. Jpn. 77, 104704 (2008).

[5] H. Koizumi, J. Supercond. Nov. Magn. 24, 1997 (2011).

[6] R. Hidekata and H. Koizumi, J. Supercond. Nov. Magn. 24, 2253 (2011).

[7] H. Koizumi, R. Hidekata, A. Okazaki, and M. Tachiki, J Supercond Nov Magn 27, 121 (2014).

[8] J. Xia, E. Schemm, G. Deutscher, S. A. Kivelson, D. A. Bonn, W. H. Hardy, R. Liang, W. Siemons, G. Koster, M. M. Fejer, et al., Phys. Rev. Lett. 100, 127002 (2008).

[9] D. LeBoeuf, N. Doiron-Leyraud, J. Levallois, R. Daou, J.-B. Bonnemaison, N. E. Hussey, L. Balicas, R. J. Ramshaw, R. Liang, D. A. Bonn, et al., Nature 450, 533 (2007).

[10] M. Fujita, H. Goka, K. Yamada, J. M. Tranquada, and L. P. Regnault, Phys Rev. B 70, 104517 (2004).

[11] Z. A. Xu, N. P. Ong, Y. Wang, T. Kakeshita, and S. Uchida, Nature 406, 486 (2000).

[12] R. Daou, J. Chang, D. LeBoeuf, O. Cyr-Choiniere, F. Laliberte, N. Doiron-Leyraud, B. J. Ramshaw, R. Liang, D. A. Bonn, W. N. Hardy, et al., Nature 463, 519 (2010).

[13] M. R. Norman, H. Ding, N. Randeria, J. C. Campuzano, T. Yokota, T. Takeuchi, T. Takahashi, T. Mochiku, K. Kadowaki, P. Guptasarma, et al., Nature 392, 157 (1998).

[14] A. Bianconi, N. L. Saini, A. Lanzara, M. Missori, T. Rossetti, H. Oyanagi, H. Yamaguchi, K. Oka, and T. Ito, Phys. Rev. Lett. 76, 3412 (1996).

[15] S. Miyaki, K. Makoshi, and H. Koizumi, J. Phys. Soc. Jpn. 77, 034702 (2008).

[16] V. J. Emery and S. A. Kivelson, Nature 374, 434 (1995).

[17] M. Hashimoto, R.-H. He, K. Tanaka, J.-P. Testaud, W. Meevasana, R. G. Moore, D. Lu, H. Yao, Y. Yoshida, H. Eisaki, et al., Nature Phys. 6, 414 (2010).

[18] Y. Kohsaka, T. Hanaguri, M. Azuma, M. Takano, J. C. Davis, and H. Takagi, Nature Phys. 8, $534(2012)$.

[19] P. W. Anderson, Phys. Rev 115, 2 (1959).

[20] M. V. Berry, Proc. Roy. Soc. London Ser. A 391, 45 (1984).

[21] J. A. Vergés, E. Louis, P. S. Lomdahl, F. Guinea, and A. R. Bishop, Phys Rev. B 43, 6099 (1991).

[22] K. Yamaji, T. Yanagisawa, M. Miyazaki, and R. Kadono, J. Phys. Soc. Jpn. 80, 083702 (2011).

[23] F. London, Phys. Rev. 74, 562 (1948). 